\title{
Comparison of arterial stiffness indices measured by the Colins and SphygmoCor systems
}

\author{
Jong-Chan Youn ${ }^{1,6}$, Jong-Youn Kim ${ }^{2,6}$, Sungha Park ${ }^{1,3}$, Jisun Kwon ${ }^{3,4}$, Hye Sun Lee ${ }^{5}$, Dong-Ho Shin ${ }^{1}$, \\ Sang-Hak Lee ${ }^{1}$, Seok-Min Kang ${ }^{1}$, Nak Hoon Son ${ }^{3}$ and Yangsoo Jang ${ }^{1,3}$
}

Arterial stiffness is a known independent predictor of cardiovascular mortality. The Colins system is an easy device and has gained widespread use, but the cutoff value for high-risk central arterial stiffness is not well established. We investigated the correlation between arterial stiffness measured by the Colins system with conventional measurements from the SphygmoCor system. Arterial pulse wave velocity (PWV) and augmentation indices (Als) were measured on a single visit using two different devices in 948 patients with hypertension or coronary artery disease. Strong positive correlations were observed for PWV values measured by the SphygmoCor and Colins systems. The Colins system measurements accurately predicted high-risk central arterial stiffness, defined as carotid-femoral PWV $\geqslant 12 \mathrm{~m} \mathrm{~s}^{-1}$, with an area under the receiver-operating characteristic curve (AUC) of 0.884 (heart-femoral PWV, hfPWV) and 0.830 (brachial-ankle PWV, baPWV) in the training set $(\boldsymbol{N}=664)$. The cutoff values, 11.18 (hfPWV) and $16.17 \mathrm{~m} \mathrm{~s}^{-1}$ (baPWV), showed good discrimination in the validation set $(N=284)$, with sensitivity of 83.3 (hfPWV) and $76.0 \%$ (baPWV), and specificity of 74.9 (hfPWV) and $82.6 \%$ (baPWV). The SphygmoCor and Colins Al systems also showed moderate positive correlation. The Colins Al system better predicted high-risk central pulse pressure as defined by pulse pressure $\geqslant 50 \mathrm{~mm} \mathrm{Hg}$ (AUC: Colins, 0.765 ; SphygmoCor, 0.692; $P=0.011$ ). Arterial stiffness measured by the Colins system showed strong positive correlation and agreement with the SphygmoCor system measurement. Cutoff values for high-risk central arterial stiffness in the Colins system need further validation in a prospective study.

Hypertension Research (2012) 35, 1180-1184; doi:10.1038/hr.2012.113; published online 26 July 2012

Keywords: arterial stiffness; augmentation index; Colins; pulse wave velocity; SphygmoCor

\section{INTRODUCTION}

Arterial stiffening results from changes in the central arteries that are accompanied by arterial dilatation, elastin degradation, increased collagen and vascular smooth muscle cell proliferation in the intima media of the arterial wall. ${ }^{1,2}$ Studies have shown that increased pulse wave velocity (PWV), an index of arterial stiffness, is an independent predictor of cardiovascular mortality in both the general population and in patients with hypertension. ${ }^{3-5}$ Carotid-femoral PWV (cfPWV), which is the ratio of the distance between the carotid artery-femoral artery and the pulse wave transit time, is considered the conventional method for measuring PWV. Studies have shown that a PWV of more than $12 \mathrm{~m} \mathrm{~s}^{-1}$ is associated with increased risk of both all-cause mortality and cardiovascular mortality. ${ }^{6,7}$ Although the measurement of heart-femoral PWV (hfPWV) and brachial-ankle PWV (baPWV) using the Colins system (VP-2000 pulse wave unit) has gained widespread popularity and usage in the clinical setting in East Asia due to its ease of use, the measurement of baPWV has been criticized because it contains components of peripheral vascular stiffness.
In addition, the algorithm for automatically calculating the path length between the arteries may be a source of error. Another limitation of the Colins system is the lack of data regarding the upper limit, above which an associated increased cardiovascular risk is seen. Therefore, we sought to determine the correlation and agreement of arterial stiffness measured by the Colins system with conventional measurements using the SphygmoCor system. We also sought to determine the cutoff value for the Colins system in predicting high-risk central arterial stiffness.

\section{MATERIALS AND METHODS}

Study population

The study population consisted of 948 subjects with hypertension and/or coronary artery disease diagnosed and treated at Severance Cardiovascular Hospital, Seoul, Korea, as part of the Yonsei Cardiovascular Genome Center cohort. The average age was $61.5 \pm 10.3$ years (range, 22-86; 681 men). In the cohort, 697 subjects had a history of hypertension and 707 subjects had a history of coronary artery disease. The subjects consented to the arterial

${ }^{1}$ Division of Cardiology, Severance Cardiovascular Hospital, Yonsei University College of Medicine, Seoul, Korea; ${ }^{2}$ Heart Center, Gangnam Severance Hospital, Yonsei University College of Medicine, Seoul, Korea; ${ }^{3}$ Cardiovascular Genome Center, Yonsei University College of Medicine, Seoul, Korea; ${ }^{4}$ Department of Cardiology, Brain Korea 21 Project for Medical Science, Yonsei University College of Medicine, Seoul, Korea and ${ }^{5}$ Department of Biostatistics, Yonsei University College of Medicine, Seoul, Korea

${ }^{6}$ These authors contributed equally to this article.

Correspondence: Dr S Park, Division of Cardiology, Severance Cardiovascular Hospital, Yonsei University College of Medicine, 50 Yonsei-ro, Seodaemun-gu, Seoul 120-752, Korea.

E-mail: shpark0530@yuhs.ac

Received 5 December 2011; revised 7 April 2012; accepted 25 May 2012; published online 26 July 2012 
stiffness measurements. PWV and augmentation index (AI) were measured on a single visit using two different devices (SphygmoCor (AtCor Medical, Sydney, NSW, Australia) and Colins systems (VP-2000 pulse wave unit; Nippon Colin Ltd, Komaki City, Japan)). Hypertension was defined as either a documented systolic blood pressure (BP) greater than $140 \mathrm{~mm} \mathrm{Hg}$ and/or a diastolic BP greater than $90 \mathrm{~mm} \mathrm{Hg}$ after at least $5 \mathrm{~min}$ of rest in a sitting position on three different visits before BP medication or current use of antihypertensive medications. Coronary artery disease was diagnosed when significant coronary artery stenosis of more than $50 \%$ was demonstrated on coronary angiography. Patients with any of the following conditions were excluded from participation: congestive heart failure, valvular heart disease, peripheral vascular disease, debilitating malignant disease, severe respiratory disease, history of inflammatory disease and/or on anti-inflammatory medications, clinically significant atrioventricular conduction disturbance, history of atrial fibrillation or other serious arrhythmia, and malignant hypertension $(>200 / 140 \mathrm{~mm} \mathrm{Hg}$ ). Peripheral vascular disease was defined as a previous diagnosis of peripheral vascular disease and/or an ankle-brachial index of less than 0.9.

This study received prior approval from the institutional ethics committee, and the procedures followed were in accordance with institutional guidelines. All patients gave informed consent before being enrolled.

\section{BP and Colins PWV measurements}

After $5 \mathrm{~min}$ of rest, brachial BP was measured three times at 2-min intervals in the dominant arm with an OMRON HEM 7080IT device (Omron Healthcare, Kyoto, Japan), which was previously validated by the British Hypertension Society. ${ }^{8}$ The average of the last two measurements was used in the statistical analyses. BP was measured before the measurement of PWV. PWV was determined by measuring the hfPWV and baPWV with a VP-2000 pulse wave unit as described previously. ${ }^{9}$ Briefly, after an overnight fast and 5-min rest, the PWV was measured in the supine position. Carotid and femoral artery pressure waveforms were recorded at the left carotid and the left femoral arteries using multi-element tonometry sensors. The electrocardiogram was monitored by electrodes on both wrists. Microphones placed at the left sternal edge in the third intercostal space were used to detect heart sounds. The hfPWV, a marker of central aortic stiffness, was calculated using the equation $\mathrm{Lhf} /(\mathrm{Thc}+\mathrm{Tcf})$, where $\mathrm{Lhf}$ is the distance from the heart to the femoral artery. Thc was defined as the time intervals between S2 and the notch of the carotid pulse wave, and Tcf was defined as the time interval between the carotid and femoral artery pulse waves. Thf, the time required for pulse waves to travel from the heart (aortic orifice) to the femoral artery, was defined as the sum of Thc and Tcf. The baPWV, a marker for both central and peripheral arterial stiffness, was calculated using the equation (D1-D2)/T, where D1 is the distance between the heart and ankle, D2 is the distance between the heart and brachium, and $\mathrm{T}$ is the transit time between the right brachial artery wave and right tibial artery wave. Carotid AI was measured from the carotid waveform obtained using a multi-element tonometer sensor as described previously. ${ }^{10}$

\section{SphygmoCor PWV measurement}

Central hemodynamics were evaluated in the sitting position after $10 \mathrm{~min}$ of rest using a commercially available radial artery tonometry device (SphygmoCor). Using a high-fidelity micromanometer (Millar Instruments, Houston, TX, USA), peripheral pressure waveforms were recorded from the radial artery at the wrist, as previously reported. ${ }^{11,12}$ The SphygmoCor system uses a generalized transfer function derived from the radial artery tonometry for obtaining the central pulse wave and central BP. Central systolic BP, diastolic BP, pulse pressure, augmentation pressure, forward wave amplitude and AI were acquired from the pulse waveform analysis. Pulse pressure was calculated as the difference between the systolic and the diastolic pressure. Augmentation pressure is the difference between the second and first systolic peak pressures, and $\mathrm{AI}$ is defined as the ratio of the augmentation pressure to the aortic pulse pressure. The cfPWV was measured as specified previously. ${ }^{13}$ Briefly, electrocardiogram and carotid/femoral pulse waves were obtained simultaneously to calculate the transit time using the foot-to-foot method. The distance traveled by the pulse wave was calculated by subtracting the sternal notch-right carotid site from right femoral site-sternal notch distances. ${ }^{13}$

\section{Statistical analysis}

Continuous variables are summarized as the mean \pm s.d. Categorical variables are summarized as a percentage of the total cohort. Continuous variables were compared using one-sample Student's $t$-tests. Pearson's correlation analysis was used for the relationships between measurements of PWV and AI. The relationship is considered to be a 'strong' association when the correlation coefficient $(r)$ is between 0.6 and 0.8 , whereas it is considered to be a 'moderate' association when $r$ is between 0.4 and 0.6. Bland-Altman analysis was used to analyze the agreement of PWV and AI measurements using the two different systems (SphygmoCor and Colins). In the Bland-Altman analysis for PWV measurements, the difference was defined by Colins PWV-SphygmoCor PWV $\left(\mathrm{m} \mathrm{s}^{-1}\right)$. As for AI measurements, the difference was defined by SphygmoCor AI-Colins AI (\%). Receiver-operating characteristic (ROC) analysis was performed to determine the accuracy of baPWV and hfPWV in predicting highrisk aortic PWV as determined using cfPWV. Youden's method was used to find an optimal cutoff point in the ROC curve to maximize the sensitivity and specificity. The accuracy of the carotid $\mathrm{AI}$ in predicting a central pulse pressure of more than $50 \mathrm{~mm} \mathrm{Hg}$ was also determined. Comparison of the ROC curve in AI was completed using the Delong method, which is a method of comparing the performance of diagnostic tests using an ROC curve. ${ }^{14}$

\section{RESULTS}

Clinical characteristics and laboratory findings

Baseline clinical characteristics and laboratory findings are summarized in Table 1. The mean value of cfPWV $\left(9.00 \pm 2.06 \mathrm{~ms}^{-1}\right)$ was

\section{Table 1 Clinical characteristics and laboratory findings of study subjects}

Characteristics Values

\section{Age (years)}

$61.5 \pm 10.3$

Male (\%)

$681(71.8)$

Hypertension (\%)

Dyslipidemia (\%)

$697(73.5)$

DM (\%)

$408(43.0)$

CAD $(\%)$

$256(27.0)$

707 (74.6)

BMI $\left(\mathrm{kg} \mathrm{m}^{-2}\right)$

$25.2 \pm 3.0$

Central SBP $(\mathrm{mm} \mathrm{Hg})$

$116.7 \pm 16.2$

Central DBP $(\mathrm{mm} \mathrm{Hg})$

$75.9 \pm 10.5$

Brachial SBP $(\mathrm{mm} \mathrm{Hg})$

$124.4 \pm 16.5$

Brachial DBP $(\mathrm{mm} \mathrm{Hg})$

$74.8 \pm 10.3$

WBC $\left(/ 10^{3} \mu \mathrm{l}\right)$

Hemoglobin $\left(\mathrm{g} \mathrm{dl}^{-1}\right)$

$6.5 \pm 1.7$

$14.2 \pm 1.4$

BUN (mg dl-1)

$17.1 \pm 6.0$

Creatinine $\left(\mathrm{mg} \mathrm{dl}^{-1}\right)$

$0.8 \pm 0.6$

Total cholesterol $\left(\mathrm{mgdl}^{-1}\right)$

$62.2+37.3$

Triglyceride $\left(\mathrm{mg} \mathrm{dl}^{-1}\right)$

$143.8 \pm 84.5$

HDL-cholesterol ( $\mathrm{mg} \mathrm{dl}^{-1}$ )

$46.6 \pm 12.4$

$87.2 \pm 32.0$

FBS (mg dl ${ }^{-1}$ )

hsCRP ( $\mathrm{mgl}^{-1}$ )

$104.0 \pm 25.4$

$1.6 \pm 4.5$

$5.2 \pm 1.4$

Uric acid (mgdl ${ }^{-1}$ )

$9.00 \pm 2.06$

PWV $\left(\mathrm{ms}^{-1}\right)$

$10.34 \pm 2.32$

$14.70 \pm 2.69$

baPWV $\left(\mathrm{ms}^{-1}\right)$

$25.7 \pm 13.8$

SphygmoCor Al (\%)

$20.8 \pm 17.8$

Abbreviations: AI, augmentation index; baPWV, brachial-ankle pulse wave velocity; BMI, body mass index; BUN, blood urea nitrogen; CAD, coronary artery disease; cfPWV, carotid-femoral pulse wave velocity; DBP, diastolic blood pressure; DM, diabetes mellitus; FBS, fasting blood sugar; HDL, high-density lipoprotein; hfPWV, heart-femoral pulse wave velocity; hsCRP, highsensitivity C-reactive protein; LDL, low-density lipoprotein; SBP, systolic blood pressure; WBC, sensitivity C-reactive
white blood cell.

Whitues are presented as $n(\%)$ or mean \pm s.d.
Value 
significantly lower than hfPWV $\left(10.34 \pm 2.32 \mathrm{~m} \mathrm{~s}^{-1} ; P<0.001\right)$ and baPWV $\left(14.70 \pm 2.69 \mathrm{~ms}^{-1} ; P<0.001\right)$. The AI measured by the SphygmoCor system $(25.7 \pm 13.8 \%)$ was significantly higher than that measured by the Colins AI system $(20.8 \pm 17.8 \%$; $P<0.001)$. Overall BP control for both central BP $(116.7 \pm 16.2 / 75.9 \pm 10.5 \mathrm{~mm} \mathrm{Hg})$ and brachial BP $(124.4 \pm 16.5 / 74.8 \pm 10.3 \mathrm{~mm} \mathrm{Hg})$ was acceptable in this cohort.

\section{Relationships of PWV using SphygmoCor and Colins}

Simple regression analysis revealed a high correlation between cfPWV and hfPWV, and between cfPWV and baPWV. The Bland-Altman analysis confirmed the acceptable agreement of PWV measurements for the SphygmoCor and Colins systems (Figure 1). To determine and validate the cutoff value for the Colins system in predicting high-risk central arterial stiffness (defined by cfPWV $\geqslant 12 \mathrm{~m} \mathrm{~s}^{-1}$ ), we randomly divided the study subjects $(N=948)$ into training $(N=664,70.0 \%)$ and validation $(N=284,30.0 \%)$ sets. Prediction of high-risk central arterial stiffness showed an area under the ROC curve (AUC) of 0.884 for hfPWV and 0.830 for baPWV in the training set (Figure 2). The cutoff values were $11.18 \mathrm{~m} \mathrm{~s}^{-1}$ for hfPWV and $16.17 \mathrm{~m} \mathrm{~s}^{-1}$ for baPWV. The cutoff values showed good discrimination in the validation set $(N=284)$, with sensitivity of 83.3 (hfPWV) and $76.0 \%$ (baPWV), and specificity of 74.9 (hfPWV) and $82.6 \%$ (baPWV; Table 2).

Relationships of AI using the SphygmoCor and Colins systems AI measured by the SphygmoCor and Colins systems also showed moderate positive correlation and good agreement (Figure 3). Interestingly, the Colins AI system showed better prediction for high-risk central pulse pressure (defined as pulse pressure $\geqslant 50 \mathrm{~mm} \mathrm{Hg}$ ) than the SphygmoCor AI system (AUC: Colins, 0.765; SphygmoCor, 0.692; $P=0.011$; Figure 4). The cutoff value was $30.5 \%$ for the SphygmoCor AI system and $24.5 \%$ for the Colins AI system. The cutoff value
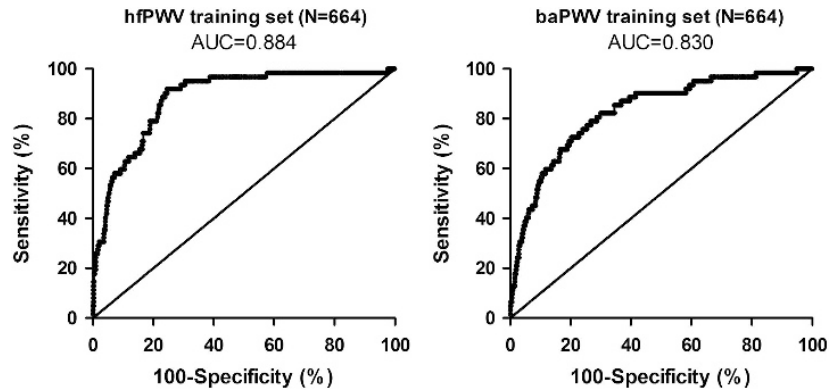

Figure 2 Receiver-operating characteristic curves for predicting high-risk central arterial stiffness as defined by cfPWV $\geqslant 12 \mathrm{~ms}^{-1}$ in the training set $(N=664)$.

Table 2 Sensitivity and specificity of PWV for predicting high-risk central arterial stiffness in the training $(N=664)$ and validation sets $(N=284)$

\begin{tabular}{ccc}
\hline $\begin{array}{c}\text { Cutoff value } \\
\left(\mathrm{ms}^{-1}\right)\end{array}$ & Sensitivity, \% & Specificity, \% \\
$(95 \% \mathrm{Cl})$ & $(95 \% \mathrm{Cl})$
\end{tabular}

\section{hfPWV}

Training $(N=664) \quad 11.18 \quad 91.9(85.2-98.7) \quad 75.4(72.0-78.9)$

Validation $(N=284)$ $83.3(68.4-98.2) \quad 74.9(69.6-80.2)$

baPWV

Training $(N=664) \quad 16.17 \quad 72.6(61.5-83.7) \quad 80.0(76.6-83.0)$ Validation $(N=284) \quad 76.0(59.3-92.7) \quad 82.6(78.0-87.2)$

Abbreviations: baPWV, baPWV, brachial-ankle PWV; cfPWV, carotid-femoral PWV; $\mathrm{Cl}$, confidence interval; hfPWV, heart-femoral PWV; PWV, pulse wave velocity. High-risk central arterial stiffness is defined by cfPWV $\geqslant 12 \mathrm{~m} \mathrm{~s}^{-1}$.
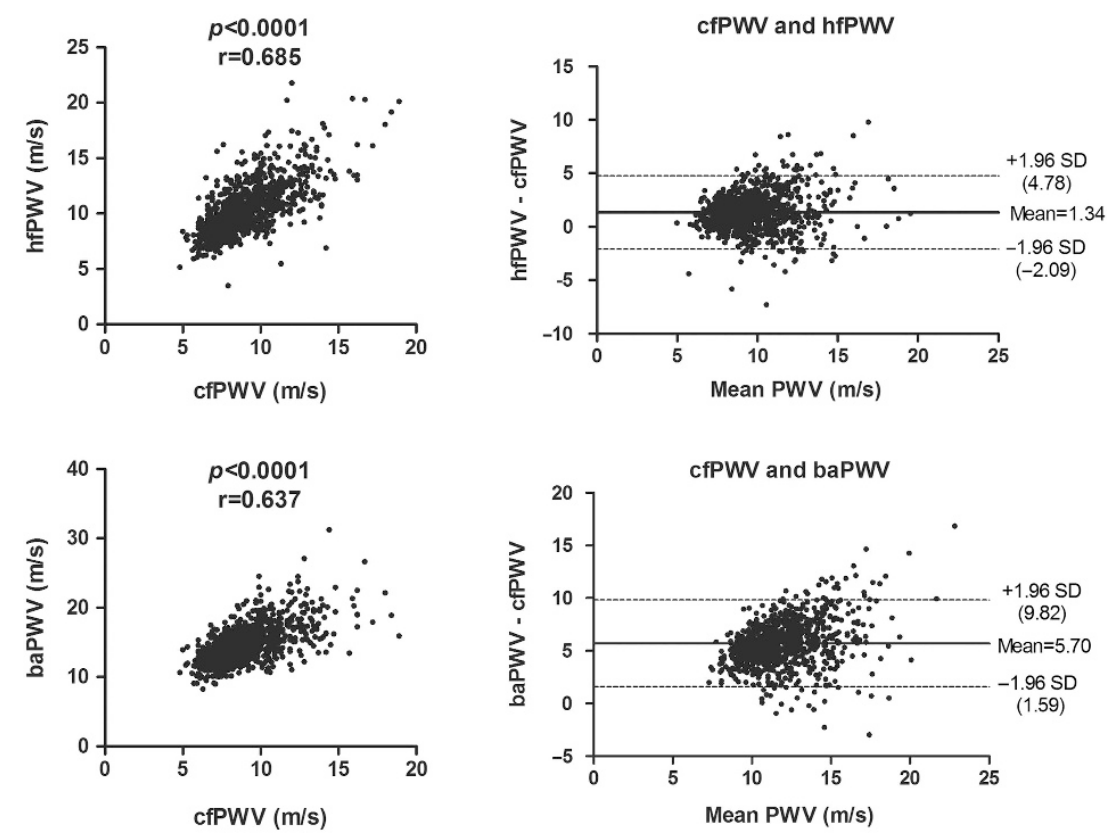

Figure 1 Relationship of PWV using SphygmoCor (cfPWV) and Colins (hfPWV and baPWV); Pearson's correlation analysis and Bland-Altman plots for pairwise comparisons. 

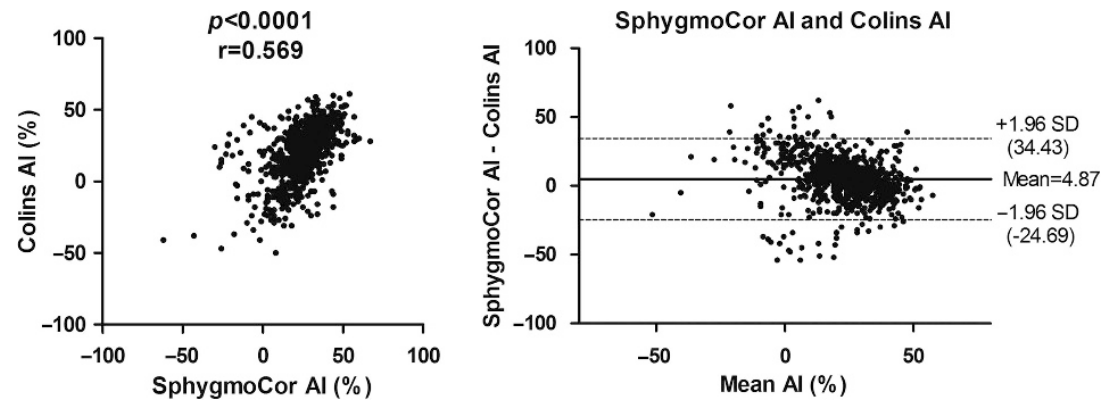

Figure 3 Relationship of Al (\%) using SphygmoCor (SphygmoCor Al) and Colins (Colins Al); Pearson's correlation analysis and Bland-Altman plots for pairwise comparisons.
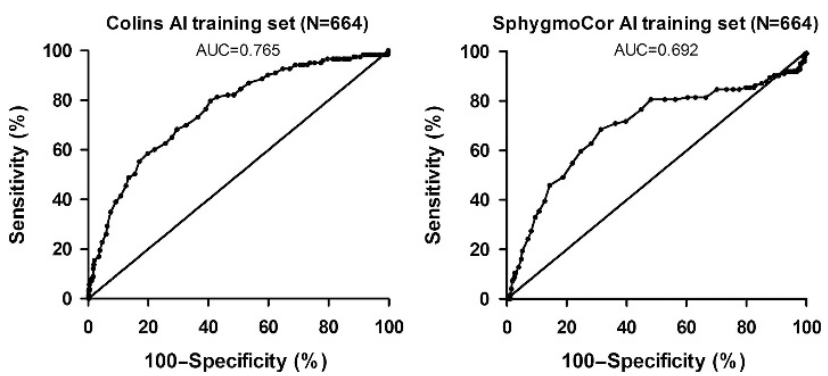

Figure 4 Receiver-operating characteristic curves for predicting high-risk central pulse pressure as defined by central pulse pressure $\geqslant 50 \mathrm{~mm} \mathrm{Hg}$ in the training set $(N=664)$.

Table 3 Sensitivity and specificity of Al for predicting high-risk central pulse pressure in the training $(N=664)$ and validation sets $(N=284)$

\begin{tabular}{ccc}
\hline Cutoff value & Sensitivity, \% & Specificity, \% \\
$(\%)$ & $(95 \% \mathrm{Cl})$ & $(95 \% \mathrm{Cl})$ \\
\hline
\end{tabular}

\section{Colins Al}

Training $(N=664)$

Validation $(N=284)$

$79.7(72.6-86.8)$

$59.4(55.2-63.5)$

SphygmoCor Al

Training $(N=664)$

30.5

$68.6(60.4-76.7)$

$68.7(64.7-72.6)$

Validation $(N=284)$

$62.3(49.2-75.3)$

$68.3(62.3-74.3)$

Abbreviations: $\mathrm{Al}$, augmentation index; $\mathrm{Cl}$, confidence interval.

High-risk central pulse pressure is defined by pulse pressure $\geqslant 50 \mathrm{~mm} \mathrm{Hg}$.

showed good discrimination in the validation set $(N=284)$, with sensitivity of 76.9 (Colins AI) and 62.3\% (SphygmoCor AI), and specificity of 63.0 (Colins AI) and 68.3\% (SphygmoCor AI; Table 3).

\section{DISCUSSION}

Despite the widespread clinical use of the Colins system for PWV measurement, there have been no data regarding the upper limit above which an association with increased cardiovascular risk is evident. We sought to determine the cutoff values of hfPWV and baPWV that most accurately predict high-risk cfPWV as measured by the SphygmoCor system. Although Tanaka et al. ${ }^{15}$ demonstrated baPWV to have good correlation with measures of central aortic stiffness, their study was limited by the fact that the index of central arterial stiffness was determined by the Colins system rather than another validated system.

In this study, there was a good correlation of both hfPWV and baPWV with conventional SphygmoCor measurements of cfPWV. The results showed that baPWV had a good predictive value for highrisk central arterial stiffness, with an AUC of 0.830 . The baPWV value of $16.17 \mathrm{~m} \mathrm{~s}^{-1}$ demonstrated a sensitivity of $72.6 \%$ and a specificity of $80.0 \%$ in the training data set. The low sensitivity of baPWV in the training set demonstrates that it may be inferior to hfPWV in predicting high-risk PWV, most likely because baPWV contains components of both central and peripheral vascular stiffness. However, as many centers use the VP-1000, which measures baPWV and not hfPWV, the AUC and accuracy of baPWV seem acceptable for assessing central arterial stiffness.

The measurement of baPWV is widespread in East Asia because of the ease of its measurement, and because, unlike femoral pulse wave acquisition, it does not require exposure of the inguinal area, which is a source of discomfort for many Asians. ${ }^{15}$ However, baPWV has been criticized because the pulse wave does not travel directly from the brachial artery to the ankle, and also because the measurement contains components of both peripheral and central arterial stiffness. ${ }^{9,15}$ The results from this data demonstrated a strong positive correlation of baPWV with cfPWV, comparable to the correlation between hfPWV, a measurement of central arterial stiffness in the Colins system, and cfPWV. The data also provide the value that could define the subset of patients at high risk for cardiovascular disease.

Recent studies have reported central BP to be an independent predictive factor for cardiovascular outcomes beyond that conferred by brachial BP. ${ }^{16,17}$ The arterial pulse wave is generated by the sum of the forward pressure wave, timing of the reflected wave and the magnitude of the reflected wave along the arterial tree. ${ }^{18}$ Arterial wave reflection occurs in the peripheral arteries due to changes in the arterial properties or in the architecture of the arterial tree. ${ }^{18}$ Reflected waves can be measured as the augmentation pressure or AI, which has emerged as a surrogate marker for cardiovascular disease. ${ }^{11,19}$ Noninvasive measurement of AI can be performed by direct pulse wave measurement of the common carotid artery or synthesized wave form of the radial artery using the generalized transfer function. We demonstrated that the SphygmoCor AI system, which uses the generalized transfer function, showed high correlation and good agreement with the Colins AI system, which directly measures the carotid artery pulse wave. However, the carotid AI measured by 
the Colins system showed better prediction for high-risk central pulse pressure than the SphygmoCor AI. The better predictive value of AI derived from the carotid tonometer may be due to the fact that measurements of AI from the carotid artery have been shown to have a good correlation with invasive measurement of AI from the aorta, whereas the accuracy of using the generalized transfer function for measuring AI has been questioned. In a study by Chen et al. ${ }^{20}$ the correlation between AI derived from applanation tonometry of the carotid artery and direct invasive measurement was 0.78, whereas the correlation between AI derived from the transfer function of the radial artery waveform and the invasive measurement ranged from 0.20 to $0.65 .^{20-23}$

This study has several potential limitations. First, our study population consisted of patients with hypertension and coronary artery disease. A strong positive correlation and good agreement between the measurements obtained by the SphygmoCor and Colins systems cannot be directly applied to a normal population or other patients with cardiovascular disease. However, hypertension and coronary artery disease are the most widespread and important disease subsets in cardiology. Similar correlation and agreement were shown by a post-hoc analysis for diabetes $(N=256,27.0 \%)$ and dyslipidemia ( $N=408,43.0 \%$; data not shown). The second limitation is the lack of invasive hemodynamic measurements of central arterial stiffness. Although it is well documented that cfPWV highly correlates with invasive measurements, the gold standard of arterial stiffness is invasive hemodynamic measurement. However, invasive measurements were not clinically indicated for the study subjects. In addition, the coronary artery disease patients were diagnosed by invasive procedure before enrollment into this study. Therefore, we could not obtain invasive hemodynamic measurements. Third, the study included patients with treated hypertension and coronary artery disease, which may confound the measurement of arterial stiffness. However, overall BP control was acceptable in this cohort, which should minimize the confounding effect of medications on the results of this study. Fourth, the cutoff values of the measurements from the Colins system were determined indirectly by comparing it to the cfPWV measurement from the SphygmoCor system rather than determining the values through a longitudinal outcome study. Although this is a limitation, we believe the results from this study have clinical merit in putting forth the previously unknown cutoff values of the Colins system that could suggest vascular organ damage.

In conclusion, arterial stiffness measured by the Colins system showed high correlation and good agreement with the SphygmoCor system. The cutoff value for high-risk central arterial stiffness in the Colins system was $11.18 \mathrm{~ms}^{-1}$ for hfPWV and $16.17 \mathrm{~m} \mathrm{~s}^{-1}$ for baPWV. These values need to be further validated in a prospective study.

\section{CONFLICT OF INTEREST}

The authors declare no conflict of interest.

\section{ACKNOWLEDGEMENTS}

This study was supported by grant 2011-0020950 from the Public Welfare \& Safety Research Program and grant 2011-0005482 from the National Research Foundation of Korea (NRF) funded by the Ministry of Education, Science and Technology, Republic of Korea.
1 Sutton-Tyrrell K, Newman A, Simonsick EM, Havlik R, Pahor M, Lakatta E, Spurgeon $\mathrm{H}$, Vaitkevicius P. Aortic stiffness is associated with visceral adiposity in older adults enrolled in the study of health, aging, and body composition. Hypertension 2001; 38: 429-433.

2 Lakatta EG, Mitchell JH, Pomerance A, Rowe GG. Human aging: Changes in structure and function. J Am Coll Cardiol 1987; 10: 42A-47A.

3 Boutouyrie P, Tropeano AI, Asmar R, Gautier I, Benetos A, Lacolley P, Laurent S. Aortic stiffness is an independent predictor of primary coronary events in hypertensive patients: A longitudinal study. Hypertension 2002; 39: 10-15.

4 Laurent S, Boutouyrie P, Asmar R, Gautier I, Laloux B, Guize L, Ducimetiere P, Benetos A. Aortic stiffness is an independent predictor of all-cause and cardiovascular mortality in hypertensive patients. Hypertension 2001; 37: 1236-1241.

5 Safar ME. Pulse pressure in essential hypertension: clinical and therapeutical implications. J Hypertens 1989; 7: 769-776.

6 Blacher J, Guerin AP, Pannier B, Marchais SJ, Safar ME, London GM. Impact of aortic stiffness on survival in end-stage renal disease. Circulation 1999; 99: 2434-2439.

7 Mancia G, De Backer G, Dominiczak A, Cifkova R, Fagard R, Germano G, Grassi G, Heagerty AM, Kjeldsen SE, Laurent S, Narkiewicz K, Ruilope L, Rynkiewicz A, Schmieder RE, Boudier HA, Zanchetti A, Vahanian A, Camm J, DeCaterina R, Dean V, Dickstein K, Filippatos G, Funck-Brentano C, Hellemans I, Kristensen SD, McGregor K, Sechtem U, Silber S, Tendera M, Widimsky P, Zamorano JL, Erdine S, Kiowski W, Agabiti-Rosei E, Ambrosioni E, Lindholm LH, Viigimaa M, Adamopoulos S, Bertomeu V, Clement D, Farsang C, Gaita D, Lip G, Mallion JM, Manolis AJ, Nilsson PM, O'Brien E, Ponikowski P, Redon J, Ruschitzka F, Tamargo J, van Zwieten P, Waeber B, Williams B. Guidelines for the management of arterial hypertension: the task force for the management of arterial hypertension of the European Society Of Hypertension (ESH) and of the European Society Of Cardiology (ESC). J Hypertens 2007; 25: 1105-1187.

8 Coleman A, Steel S, Freeman P, de Greeff A, Shennan A. Validation of the Omron m7 (hem-780-e) oscillometric blood pressure monitoring device according to the British Hypertension Society protocol. Blood Press Monit 2008; 13: 49-54.

9 Kim JS, Kang TS, Kim JB, Seo HS, Park S, Kim C, Ko YG, Choi D, Jang Y, Chung N. Significant association of c-reactive protein with arterial stiffness in treated nondiabetic hypertensive patients. Atherosclerosis 2007; 192: 401-406.

10 Sugawara J, Hayashi K, Yokoi T, Cortez-Cooper MY, DeVan AE, Anton MA, Tanaka H. Brachial-ankle pulse wave velocity: an index of central arterial stiffness? J Hum Hypertens 2005; 19: 401-406.

11 Weber T, Auer J, O'Rourke MF, Kvas E, Lassnig E, Berent R, Eber B. Arterial stiffness, wave reflections, and the risk of coronary artery disease Circulation 2004. 109: 184-189.

12 Weber T, O'Rourke MF, Ammer M, Kvas E, Punzengruber C, Eber B. Arterial stiffness and arterial wave reflections are associated with systolic and diastolic function in patients with normal ejection fraction. Am J Hypertens 2008; 21: 1194-1202.

13 Rajzer MW, Wojciechowska W, Klocek M, Palka I, Brzozowska-Kiszka M, KaweckaJaszcz K. Comparison of aortic pulse wave velocity measured by three techniques: complior, sphygmocor and arteriograph. J Hypertens 2008; 26: 2001-2007.

14 DeLong ER, DeLong DM, Clarke-Pearson DL. Comparing the areas under two or more correlated receiver operating characteristic curves: a nonparametric approach. Biometrics 1988; 44: 837-845.

15 Tanaka H, Munakata M, Kawano Y, Ohishi M, Shoji T, Sugawara J, Tomiyama H, Yamashina A, Yasuda H, Sawayama T, Ozawa T. Comparison between carotid-femoral and brachial-ankle pulse wave velocity as measures of arterial stiffness. J Hypertens 2009; 27: 2022-2027.

16 Roman MJ, Devereux RB, Kizer JR, Okin PM, Lee ET, Wang W, Umans JG, Calhoun D, Howard BV. High central pulse pressure is independently associated with adverse cardiovascular outcome the strong heart study. J Am Coll Cardiol 2009; 54: 1730-1734.

17 Pini R, Cavallini MC, Palmieri V, Marchionni N, Di Bari M, Devereux RB, Masotti G, Roman MJ. Central but not brachial blood pressure predicts cardiovascular events in an unselected geriatric population: The ICARe Dicomano Study. J Am Coll Cardiol 2008, 51: 2432-2439.

18 O'Rourke MF, Adji A. An updated clinical primer on large artery mechanics: Implications of pulse waveform analysis and arterial tonometry. Curr Opin Cardiol 2005; 20: 275-281

19 Nurnberger J, Keflioglu-Scheiber A, Opazo Saez AM, Wenzel RR, Philipp T, Schafers RF. Augmentation index is associated with cardiovascular risk. J Hypertens 2002; 20: 2407-2414.

20 Chen $\mathrm{CH}$, Ting CT, Nussbacher A, Nevo E, Kass DA, Pak P, Wang SP, Chang MS, Yin FC. Validation of carotid artery tonometry as a means of estimating augmentation index of ascending aortic pressure. Hypertension 1996; 27: 168-175.

21 Segers P, Qasem A, De Backer T, Carlier S, Verdonck P, Avolio A. Peripheral 'oscillatory' compliance is associated with aortic augmentation index. Hypertension 2001; 37 1434-1439.

22 Hope SA, Meredith IT, Cameron JD. Effect of non-invasive calibration of radial waveforms on error in transfer-function-derived central aortic waveform characteristics. Clin Sci (Lond) 2004; 107: 205-211.

23 Segers P, Rietzschel E, Heireman S, De Buyzere M, Gillebert T, Verdonck P, Van Bortel $\mathrm{L}$. Carotid tonometry versus synthesized aorta pressure waves for the estimation of central systolic blood pressure and augmentation index. Am J Hypertens 2005; 18: 1168-1173. 